\title{
Benthic boundary mixing and resuspension induced by internal seiches
}

\author{
M. Gloor, A. Wüest \& M. Münnich \\ Environmental Physics, Institute for Aquatic Sciences and Water Pollution Control, ETH-Zürich, c/o \\ EAWAG, CH-8600 Dübendorf, Switzerland
}

Key words: boundary mixing, nepheloid layer, resuspension, internal seiches, vertical modes, particles

\begin{abstract}
The effect of internal seiches on horizontal hypolimnetic bottom currents and on the stationary wellmixed benthic boundary layer (BBL) induced by these currents was studied for 2 weeks in a small prealpine lake using thermistor strings, an acoustic current meter and a CTD $(\mathrm{C}$ : conductivity, T: temperature, $\mathrm{D}$ : depth) equipped with a transmissometer. 150 profiles of temperature, conductivity and transmissivity taken during two days clearly indicate the existence of a well-mixed BBL 2 to $7 \mathrm{~m}$ thick. This is the result of intense mixing in the zone of high shear above the sediment associated with seiching motion. The concentration of suspended or resuspended particles, mainly of organic nature, within the $\mathrm{BBL}$, was 2 to 4 times greater than that measured directly above the BBL. Resuspension is thought to be associated rather with high-frequency burst-like currents with measured speeds ranging up to 7 $\mathrm{cm} \mathrm{s}^{-1}$ than with the average bottom current specd of about $2 \mathrm{~cm} \mathrm{~s}^{-1}$.
\end{abstract}

\section{Introduction}

Bottom layers containing high concentrations of particulate matter, so-called nepheloid layers, are often observed in aquatic systems. This is especially true in the ocean, where sporadically intense bottom currents due to geostrophic or thermohaline circulation occur. Pak \& Zaneveld (1983), for instance, reported nepheloid layers characterized by decreasing transmissivity in the continental rise off Nova Scotia. These thick layers are due to so-called benthic storms, which typically last for 2 to 5 days with an intensity which is surprisingly high for such great depths. During these storms they found concentrations of suspended particles of up to $12 \mathrm{mg} \mathrm{l}^{-1}$. Furthermore their observations indicate the existence of burst-like events resulting in a decrease in transmissivity. In lakes, where hypolimnetic currents are generally less energetic than deep water currents in the ocean, nepheloid layers are usually thinner and less turbid. Sandilands \& Mudroch (1983) reported a nepheloid layer in Lake Ontario which was found to exist permanently at all depths below $60 \mathrm{~m}$. They found a typical suspended particle concentration of $0.8 \mathrm{mg}^{-1}$. Although the origin of the nepheloid layer is not clear, largescale motion of bottom water is expected to be responsible for its existence. Even in small lakes, there is evidence of a thin nepheloid-like layer with increased particle concentrations above bottom sediments (Bloesch \& Uehlinger, 1986).

Here we report on the role of internal seiche motion on hypolimnetic bottom currents, on the build-up of a well-mixed bottom boundary layer and on the subsequent distribution of suspended particles above the sediment. Two types of standing waves, so-called seiches, are ubiquitous in 
lakes and reservoirs: high frequency surface seiches with small vertical amplitudes (centimeters) and low frequency internal seiches with large amplitudes (meters). Since both classes of seiches are always accompanied by horizontal currents in the hypolimnion, they must be regarded as a source of benthic boundary mixing and resuspension. The flow-induced bottom stress on the sediment may resuspend sediment particles if this stress exceeds a certain threshold value which depends on the density and size of the particles (Shields, 1936). Since the velocity of hypolimnetic currents vanishes at the sediment-water interface, the seiching motion leads to high shear and consequently to intense mixing above the sediment (Thorpe, 1988). As a result, resuspended particles should undergo fast mixing within this zone, the so-called benthic boundary layer $(\mathrm{BBL})$ which we define as the region of reduced gradients above the sediment-water interface.

The BBL, the existence of which is often revealed by fine-scale high-resolution CTD profiles, is the main location not only of the transport and mixing processes affecting dissolved and particulate compounds in lake hypolimnia, but also of biogeochemical transformations. These processes are favored by the proximity of sediment particles (e.g. mineralization of biomass: Jørgensen \& Des Marais, 1990) and strong redox gradients in the adjacent water above, or in the sediment below the BBL (e.g. manganese oxidation: Johnson et al., 1991; Wehrli et al., 1993). Consequently, knowledge of benthic boundary mixing and resuspension is a nccessary prerequisite for understanding the biogeochemical processes occurring in the deep-water layers of lakes.

\section{Experiment}

The experimental work was conducted between 8 and 22 May 1992 in Lake Alpnach, the smallest (surface area $4.76 \mathrm{~km}^{2}$ ) and almost separated basin of Lake Lucerne, situated in Central Switzcrland. Its shape is approximately elliptic, with major and minor axes of length $\sim 5 \mathrm{~km}$ and $\sim 1.5 \mathrm{~km}$, respectively, and a maximum depth of
$34 \mathrm{~m}$ (Fig. 1). The density stratification is strong during summer (maximum stability $\mathrm{N}^{2} \sim 1 \cdot 10^{-3}$ to $3 \cdot 10^{-3} \mathrm{~s}^{-2}$ ) and almost unaffected by dissolved solids (salinity $\sim 0.3 \%$ ).

External wind forcing is determined mainly by the surrounding topography. A plot of the wind stress distribution measured during a previous experiment in July 1989 (Fig. 1) indicates that the prevailing winds are from ENE. The wind shows a remarkably regular diurnal pattern due to the heating/cooling cycle of the adjacent mountains (Münnich et al., 1992). The major river input (Sarner Aa) is warm epilimnetic water from another lake, which consequently merges into the epilimnion of Lake Alpnach. Two minor tributaries have negligible discharges in the absence of thunderstorms. Since Lake Alpnach is separated by a $3 \mathrm{~m}$ deep sill from the rest of Lake Lucerne, the hypolimnion of Lake Alpnach is decoupled from river water and from Lake Lucerne water masses. Consequently, in the absence of floodinduced turbidity currents, wind is the only driving force possible for currents in the hypolimnion.

In order to monitor the internal seiche motion, three Aanderaa thermistor chains (Bergen, Norway), each consisting of eleven evenly-spaced sensors with a resolution of $0.023^{\circ} \mathrm{K}$ sampling at a rate of $5 \mathrm{~min}$, were installed along the major axis of the lake at sites A (length of thermistor chain: $15 \mathrm{~m}), \mathrm{B}(20 \mathrm{~m})$ and C (15 m) (Fig. 1). In addition, a Neil Brown EG\&G acoustic current meter (Cataumet, Mass., USA: resolution: $2 \mathrm{~mm} \mathrm{~s}^{-1}$ ) was deployed $1.55 \mathrm{~m}$ above the sediment at site B (the center of the lake) during the first week, and $0.7 \mathrm{~m}$ above the sediment at site $C$ during the second week

Between 11 and $13 \mathrm{May}$, profiles of conductivity, temperature and transmissivity (wavelength $=680 \mathrm{~nm}$, path length $=7.5 \mathrm{~cm}$ ) were measured with a Meereselektronik CTD profiler (Trappenkamp, Germany) at intervals of about $15 \mathrm{~min}$ for 48 hours. During the day, measurements were conducted on a rotating basis at four fixed stations (i.e. according to the scheme: 1, 2, $3,4,1, \ldots$ ) along the major lake axis, whereas during the night they were limited to site $\mathrm{B}$. The CTD was mounted on a lander to prevent the 


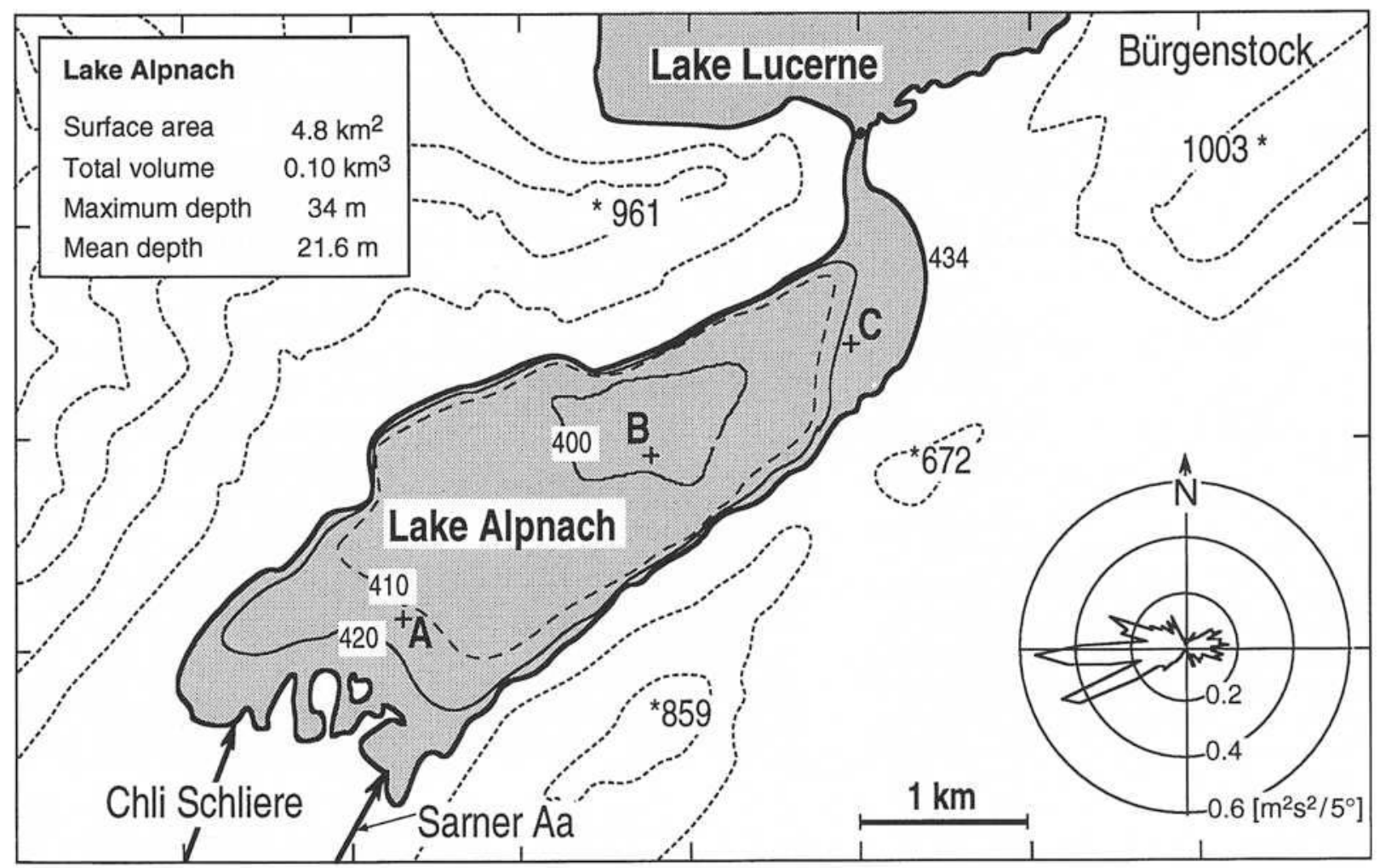

Fig. 1. Map of Lake Alpnach (a side basin of Lake Lucerne, Switzerland). The locations of the thermistor strings, labeled A (15 m), B $(20 \mathrm{~m})$ and $C(15 \mathrm{~m})$, are marked by " + '. Depth contours, lake surface elevation and the heights of nearby mountains (*) are given in $\mathrm{m}$ a.s.l. Inset: Cumulative distribution of the square of the wind speed at mooring B, measured in July 1989 (vectors shown in the wind direction). From Münnich et al. (1992).

transmissivity sensor from penetrating the sediment. Finally, using Niskin bottles, samples of suspended particles were taken close to the bottom at three sites close to $\mathrm{B}$. To prevent contamination of the samples with sediment particles, the height of the Niskin bottles above the lake bottom (about $0.3 \mathrm{~m}$ ) was checked using an echosounder and the CTD profiles were always taken after sampling. From these samples, particle dry weight was determined using pre flushed and pre dried Cellulose Acetate filters $(0.45 \mu \mathrm{m})$ and the particle size distribution was analysed using a Grimm laser particle counter (Ainring, Germany) adapted at EAWAG. The relationship between dry weight and transmissivity was established by using linear regression (cf. e.g. Baker \& Lavelle, 1984).

\section{Observations}

\section{Internal seiches and bottom currents}

Excitation of the first vertical seiche mode is very common in lakes: wind stress forces epilimnetic water towards the downwind end of the lake, thus giving rise to a horizontal pressure gradient, which in turn accelerates hypolimnetic water towards the upwind end. This current pattern is characteristic of the first vertical seiche mode $1 \mathrm{~V}$ (Fig. 2a). In Lake Alpnach, however, the second vertical seiche mode $2 \mathrm{~V}$, which is rarely reported in lakes (LaZerte, 1980; Wiegand \& Chamberlain, 1987; Münnich et al., 1992), is often predominant during summer. This mode may be understood with the help of a three layer density 
First vertical seiche mode $1 \mathrm{~V}$

a)

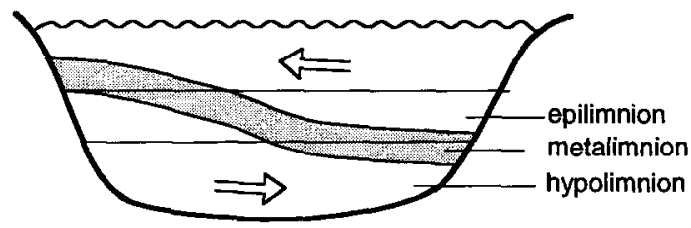

Second vertical seiche mode $2 \mathrm{~V}$

b)

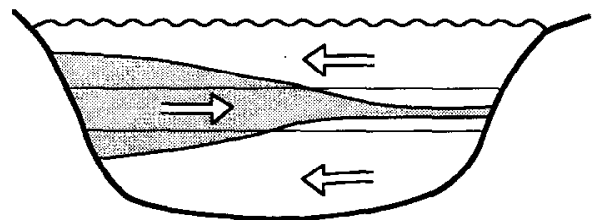

Fig. 2. Schematic illustration of the vertical structure of the displacement of (a) the first vertical (1V), and (b) the second vertical $(2 \mathrm{~V})$ seiche modes. The first vertical mode can be described by two layers, whereas for the second vertical mode a three layer model is necessary. Both seiches are first horizontal modes. The arrows indicate the flow directions after the maximum excursion of the layers. model. The middle layer is squeezed out periodically from one end of the lake to the other by the upper and lower layers (Fig. 2b). The dominance of the second vertical mode in Lake Alpnach is due to the proximity of this seiche period to the one-day period of the external wind forcing. The higher horizontal modes of both of these vertical modes can scarcely be detected in Lake Alpnach and will be disregarded here.

Figure 3 shows the displacement of the $7.5^{\circ} \mathrm{C}$ and $11^{\circ} \mathrm{C}$ isotherms at site $\mathrm{A}$ as well as the velocity of the bottom current along the major lake axis at sites B and C. Isotherm depth series were computed from the thermistor data by linear interpolation. Three different episodes of the oscillatory isotherm motion may be distinguished:

1. From 8-11 May, the upper and lower isotherms oscillate at the same frequency, corresponding to a period slightly greater than $1 \mathrm{~d}$; in addition the directions of the vertical motion

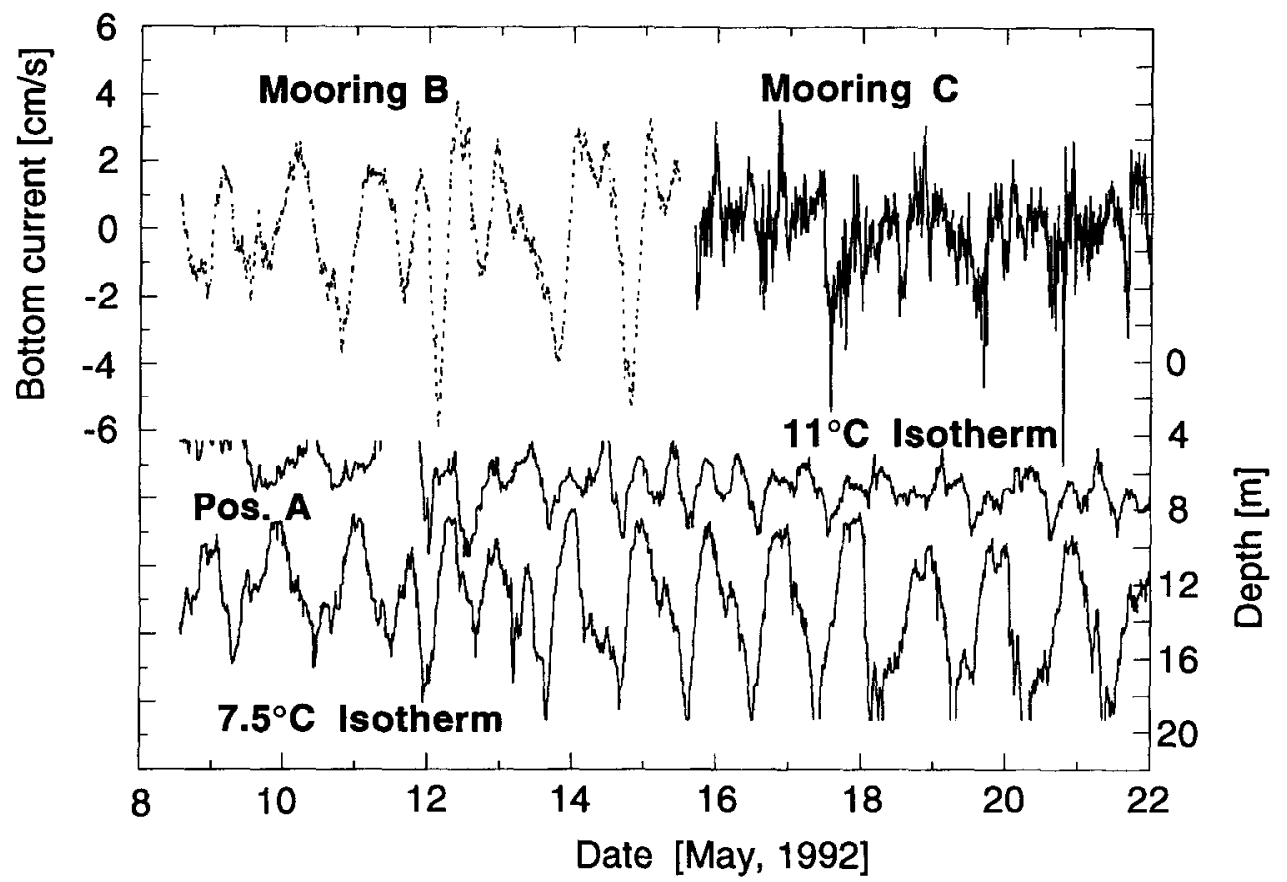

Fig. 3. Above: The component of the bottom current along the major axis of the lake at moorings B and C. Positive values indicate current flowing towards ENE. Sampling rates at station B and station C were $5 \mathrm{~min}$ and $1 \mathrm{~min}$, respectively. The graph of the currents at site $\mathrm{C}$ have been smoothed using a $5 \mathrm{~min}$ running mean. Below: Time series of the depths of two selected isotherms at mooring A. (Since temperature was measured from 4.5 to $19.5 \mathrm{~m}$ depth at station $\mathrm{A}$, isotherms outside this vertical range are not available.) 
are negatively correlated (Fig. 3), which is typical of the $2 \mathrm{~V}$ seiche mode.

2. From 12-14 May, on the other hand, the directions of motion are positively correlated and the periods of oscillation are about $0.5 \mathrm{~d}$, implying excitation of the $1 \mathrm{~V}$ seiche mode.

3. From 14-22 May, the directions of motion of the two layers are not correlated, the lower isotherm oscillating with a period of about $1 \mathrm{~d}$ and the upper with a period of about $0.5 \mathrm{~d}$. For this last episode the internal motion is interpreted as a superposition of the responses of the $1 \mathrm{~V}$ and $2 \mathrm{~V}$ modes to the daily forcing.

The velocity of the bottom current along the major axis of the lake at site $B$ is highly correlated with the displacement of the $7.5^{\circ} \mathrm{C}$ isotherm at site $\mathrm{A}$ (Fig. 3). The shape of the power spectra of the bottom current and of the isotherm displacement are nearly identical, and both have two maxima at periods of $13 \mathrm{~h}$ and $28 \mathrm{~h}$, the periods of the $1 \mathrm{~V}$ and $2 \mathrm{~V}$ modes, respectively (Fig. 4a). Bottom current and isotherm displacement are highly coherent $(>0.95)$ at both mode periods (13 and $28 \mathrm{~h}$ ), and the corresponding phases between the two time series are about $90^{\circ}$ (Fig. 4b). Indeed, one would expect both modes to be associated with a phase shift of $90^{\circ}$, since maximum displacement of the isopycnal surfaces at one end of the lake should be accompanied by zero current speed in the hypolimnion, and vice versa. The time lag corresponding to a phase of $90^{\circ}$ can be seen quite well in Fig. 3 for the episode of 8 to 12 May. From these consistencies we can conclude that the bottom current speed, which is averaging to $2 \mathrm{~cm} \mathrm{~s}^{-1}$ for both stations $\mathrm{B}$ and $\mathrm{C}$, is associated only with the internal seiches.

Although the correlation between the bottom current and the displacement of the $7.5^{\circ} \mathrm{C}$ isotherm at $A$ is well for both stations $C$ and $B$, the current velocity at station $\mathrm{C}$ exhibits more highfrequency variation than at station $B$. This fact can neither be attributed to the higher sampling rate nor to artificial spikes in the record. These high velocities correspond rather to high-frequency burst-like events with a duration of approximately fifteen minutes and maximum veloci- ties of $7 \mathrm{~cm} \mathrm{~s}^{-1}$. These patterns might reveal horizontal eddies originating from irregularities of lake bottom topography or from barotropic wind forcing. Burst-like events may be of particular interest here, since they have been observed to cause episodic resuspension in lakes (Lemmin \& Imboden, 1987).

\section{Benthic boundary layer}

Figure 5 shows three out of 150 profiles of temperature and of concentration of suspended matter measured on 12 May at site B near the lake bottom. The temperature profiles (Fig. 5a) indicate clearly the existence of a BBL 2 to $7 \mathrm{~m}$ thick. In most cases, the BBL was not perfectly mixed; however, the temperature gradients within the BBL $\left(\approx 0.01 \mathrm{~K} \mathrm{~m}^{-1}\right)$ were at least one order of magnitude smaller than those just above it ( $>0.1 \mathrm{~K} \mathrm{~m}^{-1}$ ).

With regard to thickness and structure of the BBL, the threc profiles illustrated in Fig. 5 are representative of the entire set of profiles, showing that the $\mathrm{BBL}$ persisted during the whole 48 hour period. The time, $t$, required to build up a mixed bottom layer of height, $h$, by continuous erosion of a background density stratification with a stability, $\mathrm{N}^{2}$, is given by $t \approx 5 / 3 \cdot\left(\mathrm{N}^{2} \mathrm{~h}^{3}\right) /\left(\mathrm{u}^{* 3} / \kappa\right)$ (Imboden \& Wüest, 1994), where $u^{*}$ is the friction velocity and $\kappa=0.41$ is the von Kármán constant. For the measured local background stability $\mathrm{N}^{2} \approx 2 \cdot 10^{-5} \mathrm{~s}^{-2}$, a typical bottom velocity $u \approx 2 \mathrm{~cm} \mathrm{~s}^{-1}$ (Fig. 3 ) and a BBL thickness $\mathrm{h} \approx 5 \mathrm{~m}$ (Fig. 5), we find the required time scale, $t$, to be more than one month. Hence the variations in boundary layer thickness, as shown at site B (Fig. 5), cannot be the result of repetitive erosion induced by mixing, but must be due to the seiching motion, the BBL itself being in a quasi-steady state.

\section{Resuspension}

The transmissivity profiles show that the concentration of suspended particles in the BBL was 2 


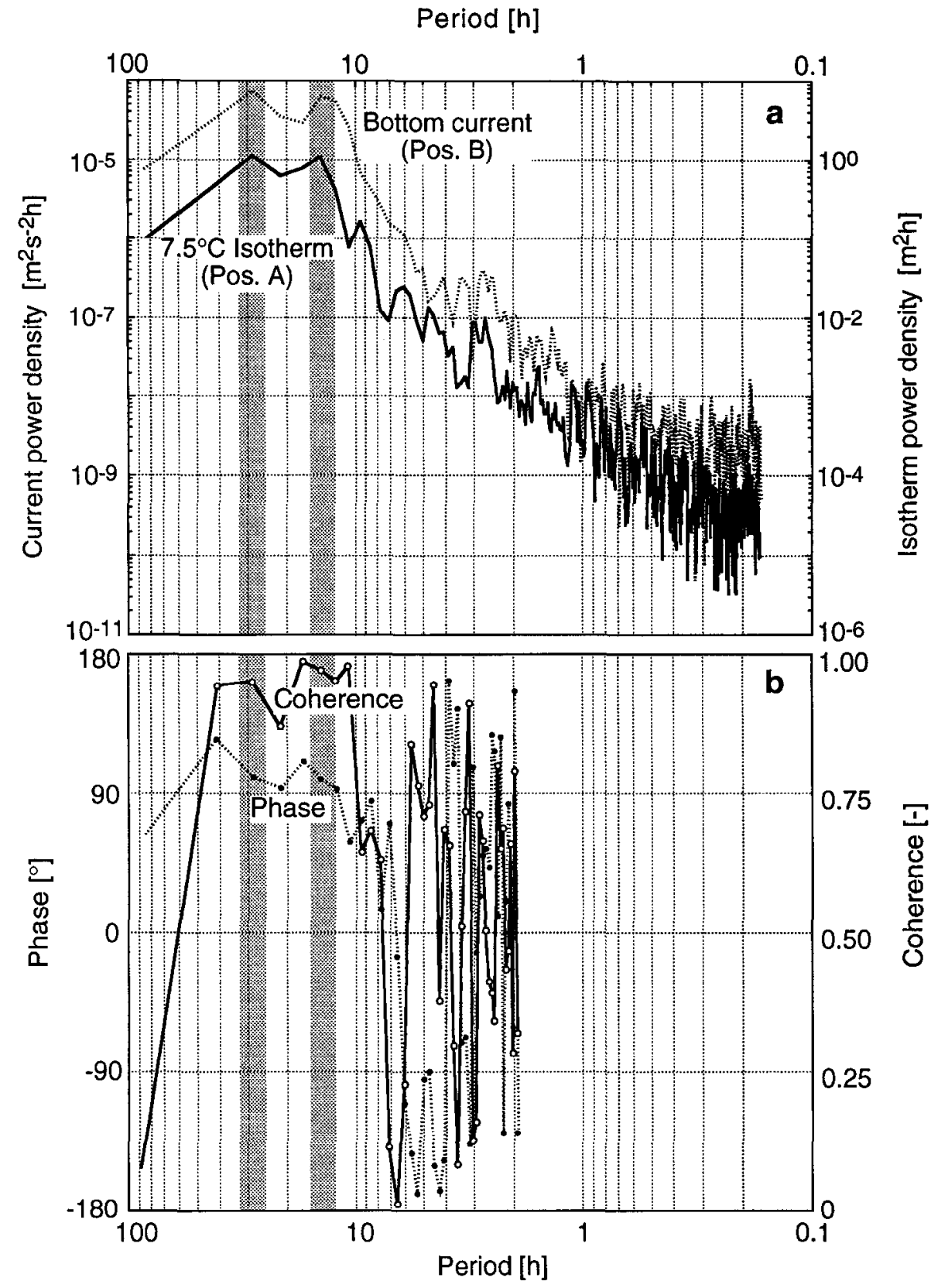

Fig. 4. a: Power density spectra of the $7.5^{\circ} \mathrm{C}$ isotherm at mooring $\mathrm{A}$ and of the bottom current at mooring B. b: Coherence and phase spectra of bottom current and isotherm depth. A phase angle of $90^{\circ}$ means that the $7.5^{\circ} \mathrm{C}$ isotherm at mooring $\mathrm{A}$ lags the bottom current time series at mooring B by a quarter period, which is exactly the value expected for first horizontal seiche modes. The vertical shading represents the periodicities of the two vertical modes discussed in the text $(28 \mathrm{~h}, 13 \mathrm{~h})$.

to 4 times greater than that directly above the BBL (Fig. 5b). Like the temperature profiles, the concentration profiles illustrated in Fig. $5 \mathrm{~b}$ are representative of all the other 150 concentration profiles. Since we can exclude lateral particle input from external sources as discussed above, and since we can reasonably assume, that the particle settling velocity remains constant within 

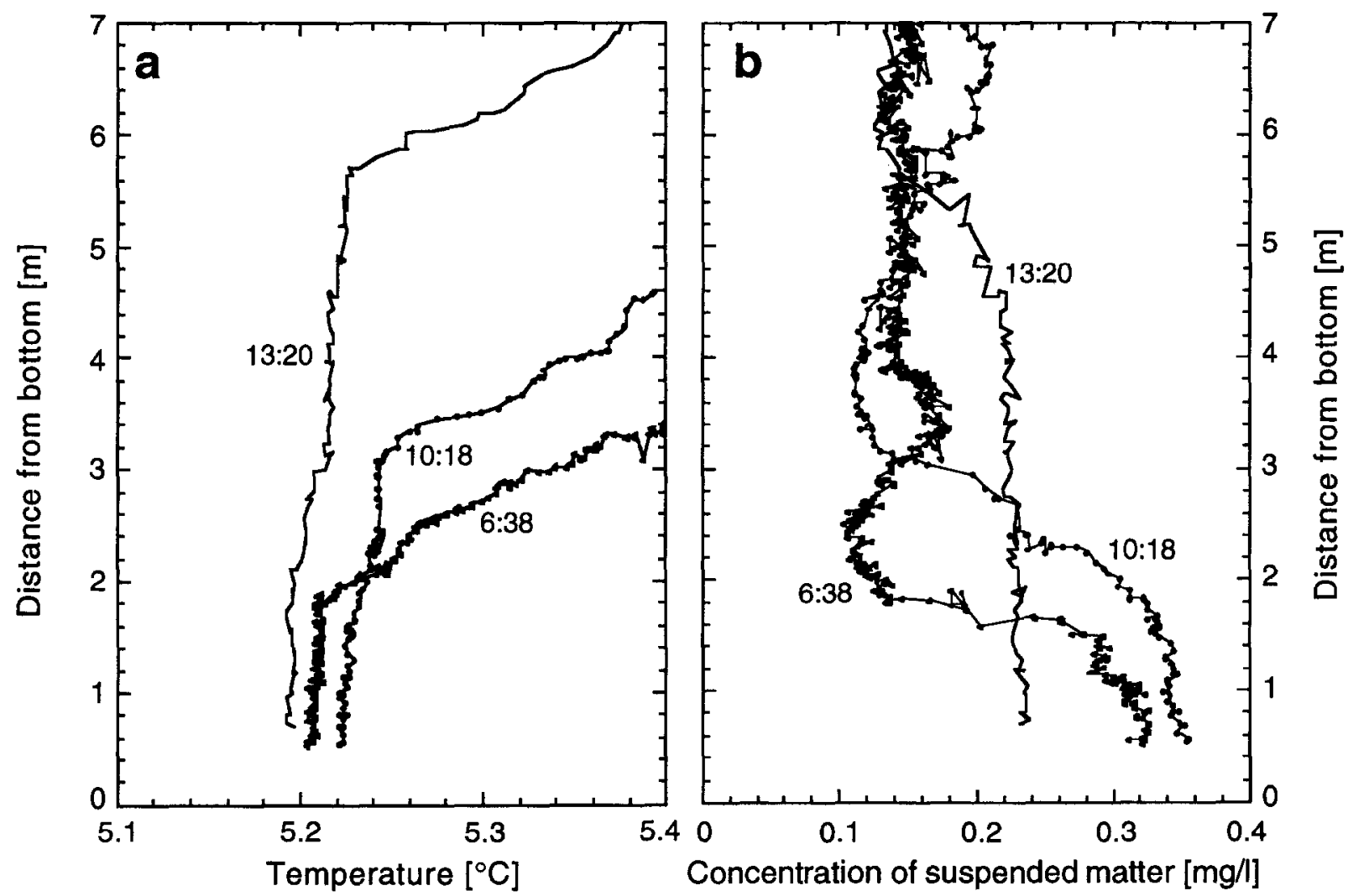

Fig. 5. Three examples of profiles through the benthic boundary layer at mooring B. Temperature (a) and suspended matter concentration (b) indicate well-mixed bottom layers. The profiles are labeled with the times of May 12, 1992, when the profiles were measured.

the deep-water body, the decrease of particle concentration with increasing distance from the sediment indicates that the source of particles must have been below the BBL; i.e. that the particles were resuspended from the sediment.

The particle distribution within the BBL is not as homogeneous as the temperature. The decrease in particle concentration in the upper part of the $\mathrm{BBL}$ is a consequence of the growing importance of the process of Stokes' settling with increasing distance from the sediment due to the decrease of turbulent diffusion (Dewey \& Crawford, 1988). In the lower part of the BBL, however, the time scale for turbulent diffusion, $t_{\text {diff }} \approx h^{2} / K_{z}$, (h: $\mathrm{BBL}$ thickness, $K_{z}$ : vertical eddy diffusivity) is much smaller than the time scale for Stokes settling, $t_{\text {settling }} \approx h / w_{s},\left(w_{s}\right.$ : settling velocity); hence in this region particles, almost unaffected by gravity, behave like a dissolved compound and are consequently homogeneously mixed.

The three particle samples taken $0.3 \mathrm{~m}$ above the bottom have an average dry weight of 0.47 $\mathrm{mg}^{-1}$. Particle concentrations (for particle diameters exceeding the lower detection limit of $0.3 \mu \mathrm{m}$ ) ranged from $1.5 \cdot 10^{8}$ to $1.9 \cdot 10^{8}$ particles $1^{-1}$, with $90 \%$ of these particles smaller than $0.8 \mu \mathrm{m}$ in diameter. Assuming the particles to be spherical, their specific volume and specific surface area would be $\sim 2.4 \cdot 10^{9}(\mu \mathrm{m})^{3} 1^{-1}$ and $\sim 0.8 \cdot 10^{9}(\mu \mathrm{m})^{2} 1^{-1}$, respectively. The large specific surface area of the particles emphasizes the importance of resuspension for the mineralization process at the sediment. 


\section{Discussion}

First, we consider the nature of the suspended particles. Assuming that the density of organic particles in Lake Alpnach exceeds that of water by about $5 \%$ (J. Bloesch, pers. comm.), we find from the total specific volume of suspended particles that about $95 \%$ of the volume of the suspended particles must be of organic origin.* This conclusion is supported by the cxtended Shields diagram shown in Fig. 6.

From this diagram, the experimentally determined threshold for resuspension of non-cohesive particles may be inferred (Miller et al., 1977). The abscissa of the diagram is the non-dimensional particle size, defined as the Reynolds number $\operatorname{Re}^{*}=\left(u^{*} D / v\right)$, which is proportional to the ratio of the particle size to the thickness of the viscous sublayer (for the definition of the various quantities consult the legend of Fig. 6). The ordinate is the non-dimensional shear stress $\mu u^{* 2} /$ $\left(\left(\rho_{\mathrm{p}}-\rho\right) g D\right)$, which expresses the ratio of the lifting force of the bottom stress to the force of gravity on the particle. For $\left(\mathrm{Re}^{*}, \theta\right)$-values lying above the upper curve, $\theta_{\text {high }}$, particle transport is likely, whereas it is unlikely for ( $\left.\mathrm{Re}^{*}, \theta\right)$-values lying below the lower curve, $\theta_{\text {low }}$ (Fig. 6). $\left(\operatorname{Re}^{*}, \theta\right)$ values between the two curves correspond to critical values for initiation of particle transport (Miller et al., 1977). The original diagram by Shields (1936), which was established for values of $\operatorname{Re}^{*}>1$, has been extended by Miller et al. (1977) down to $\mathrm{Re}^{*}=0.05$.

To test for resuspension, the non-dimensional stress $\theta$ as a function of particle diameter $D$ was compared with the extended Shields curve of Fig. 6 for two current velocities and both organic and inorganic particles. Density differences of $\left(\rho_{\mathrm{p}}-\rho\right) / \rho=1.65$ (typical for inorganic sediment particles) and $\left(\rho_{\mathbf{p}}-\rho\right) / \rho=0.05$ (upper limit for

\footnotetext{
* The volumetric fraction of particles of organic origin, $\alpha$, is given by the mass equation: $P_{\text {tot }}=v_{\text {tot }} \cdot\left[\alpha \rho_{\text {org }}+(1-\alpha) \rho_{\text {inorg }}\right]$, with $P_{\text {tot }}$ : observed particle concentration (dry weight), $v_{10 t}$ : the specific particle volume, $\rho_{\text {org }}$ : density of organic particles, $\rho_{\text {inorg }}:$ density of inorganic particles. For the above reported values $P_{\mathrm{tot}}=0.47 \mathrm{mg} \mathrm{l}^{-1}, \quad v_{\mathrm{tot}}=2.4 \mathrm{~mm}^{3} \mathrm{I}^{-1}$, $\rho_{\text {org }}=0.05 \mathrm{~kg} \mathrm{l}^{-1}$ and $\rho_{\text {inorg }}=2.65 \mathrm{~kg} \mathrm{l}^{-1}$ we find $\alpha \approx 0.95$.
}

organic particles) were assumed. The current velocities used were $2 \mathrm{~cm} \mathrm{~s}^{-1}$, the root mean square of the bottom current speed over the first week (Fig. 3), and $7 \mathrm{~cm} \mathrm{~s}^{-1}$, the peak velocity during burst-like events. Corresponding friction velocities $u^{*}$, calculated by applying the relationship $u^{*}=\left(C_{1 \mathrm{~m}} u^{2}\right)^{1 / 2}$ with a drag coefficient $C_{1 \mathrm{~m}}$ of $1.5 \cdot 10^{-3}$ (Elliott, 1984), were $0.8 \cdot 10^{-3} \mathrm{~m} \mathrm{~s}^{-1}$ and $2.7 \cdot 10^{-3} \mathrm{~m} \mathrm{~s}^{-1}$, respectively.

From the cxtended Shields diagram, critical values of $\theta$ for $\mathrm{Re}^{*}$ ranging from 0.05 to 3000 may be inferred. In Fig. 6, only the lower end of the diagram is shown, since in our situation $\mathrm{Re}^{*}$ values are usually lower than 0.05 . Below $\mathrm{Re}^{*}=0.05$, however, Shields scaling is probably inappropriate. Indeed, remembering that $\mathrm{Re}^{*}$ is proportional to the ratio of particle size to viscous sublayer thickness, particle resuspension might be expected to become independent of the thickness of the viscous sublayer at low Reynolds numbers. Since particles may be assumed to remain in suspension for considerable time only if the vertical velocity of eddies, which is of the same order as the friction velocity $u^{*}$, significantly exceeds the Stokes' settling velocity, $w_{\mathrm{s}}$, a more appropriate criterion for resuspension at low Reynolds numbers might be $w_{\mathrm{s}} / u^{*} \ll 1$. Using Stokes' law, which is applicable for $\mathrm{Re}^{*}<0.05$ (cf. e.g. Yalin, 1977), we find for spherical particles $w_{\mathrm{s}} / u^{*}=\mathrm{Re}^{*} /(18 \theta)$. Since the Shields diagram is a log-log-plot, lines of constant $w_{\mathrm{s}} / u^{*}$ follow lines with slope +1 . Consequently, we expect the critical range for resuspension in Shields diagram to bend downwards at low Reynolds numbers as indicated in Fig. 6 (dotted lines). Experimental results on the critical range of particle resuspension with diameter $>0.1 \mu \mathrm{m}$ given by Vanoni (1964) indicate that this threshold exhibits a large scatter but nevertheless support the idea of a decreasing tendency of the critical range.

A further difficulty with the use of the Shields diagram in our situation is that this diagram was established by experimenting with non-cohesive particles only. For cohesive particles the critical range for resuspension would be expected to be shifted to $\theta$-values larger than for non-cohesive particles. 


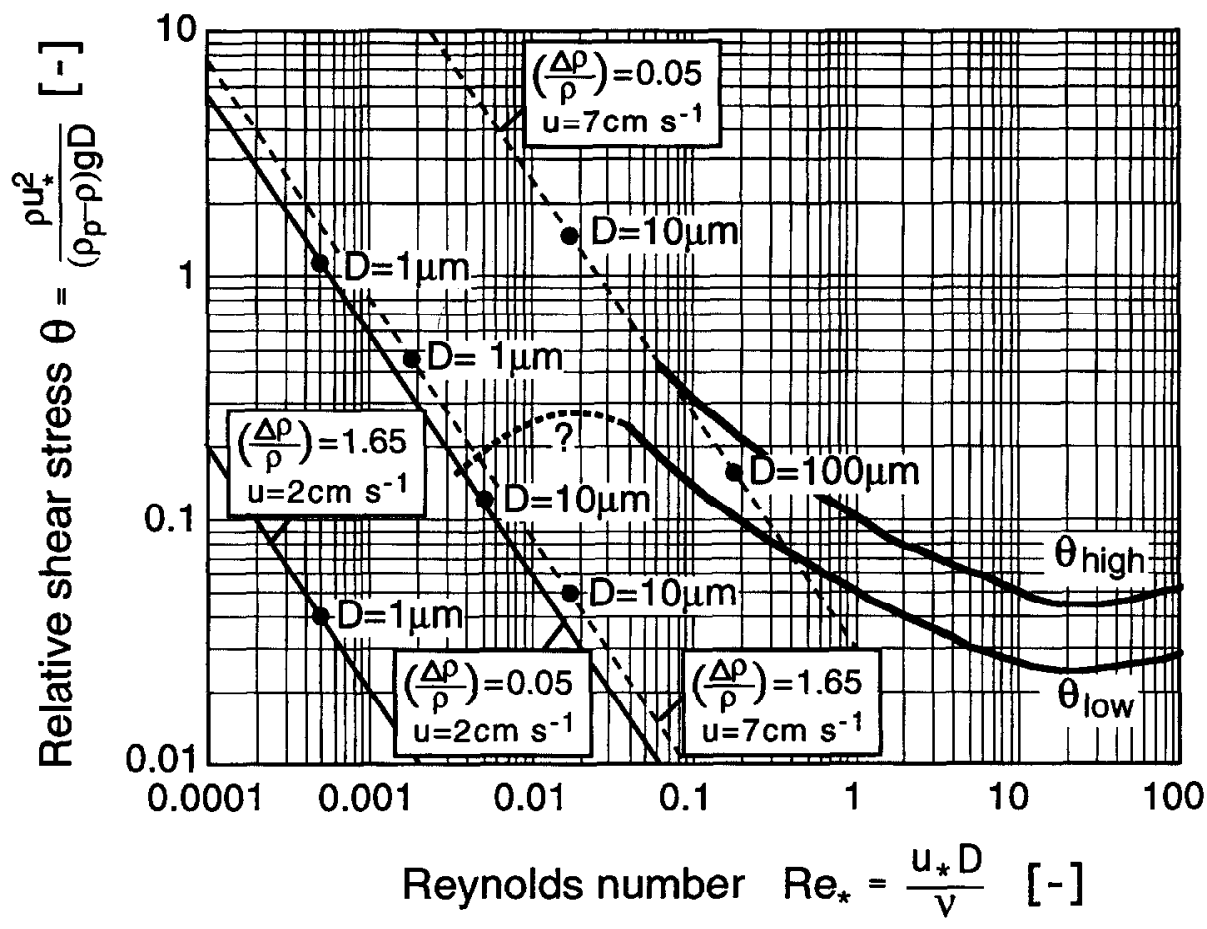

Fig. 6. Extended Shields diagram, redrawn from Miller et al. (1977). The quantities upon which the Shields diagram is based are the following: the current-induced stress on the sediment surface, $\rho u^{* 2}\left[\mathrm{~N} \mathrm{~m}^{-2}\right]$; the density of water, $\rho\left[\mathrm{kg} \mathrm{m}{ }^{-3}\right] ;$ the density of the suspended particles, $\rho_{\mathrm{p}}\left[\mathrm{kg} \mathrm{m}^{-3}\right]$; the friction velocity, $u^{*}=(\tau / \rho)^{1 / 2}\left[\mathrm{~m} \mathrm{~s}^{-1}\right]$; the acceleration of gravity, $\mathrm{g}\left[\mathrm{m} \mathrm{s}^{-2}\right]$; the particle diameter, $\mathrm{D}[\mathrm{m}]$; and the kinematic viscosity, $\nu=1.5 \cdot 10^{-6} \mathrm{~m}^{2} \mathrm{~s}^{-1}$ (at $T=5^{\circ} \mathrm{C}$ ). $\operatorname{Re}^{*}$ and $\theta$ are non-dimensional expressions for particle size and bottom stress, respectively.

The solid lines represent the upper $\left(\theta_{\text {high }}\right)$ and lower $\left(\theta_{\text {low }}\right)$ limits of the critical range of $\left(\operatorname{Re}^{*}, \theta\right)$-values for onset of sediment movement, based on empirical data. For $\left(\operatorname{Re}^{*}, \theta\right)$-values lying above the upper curve, $\theta_{\text {high }}$, particle transport is likely, whereas it is unlikely for $\left(\mathrm{Re}^{*}, \theta\right)$-values lying below the lower curve, $\theta_{\text {low }}$. The dotted part of the $\theta_{\text {low }}$-curve at $\mathrm{Re}^{*}<0.04$ indicates a suggestion for the adequate extrapolation of the critical range for small Re*-values. The black and dashed lines of slope -1 represent values of $\operatorname{Re}^{*}$ and $\theta$ calculated for $u=2 \mathrm{~cm} \mathrm{~s}^{-1}$ and $u=7 \mathrm{~cm} \mathrm{~s}^{-1}$, respectively, and relative density differences of 1.65 (mineral particles) and 0.05 (organic particles), respectively.

Although the extended Shields diagram does not cover the required range of low Reynolds numbers (Fig. 6), it indicates that the average long-term currents of the internal seiche motion may be too slow to initiate particle resuspension. For burst-like events, however, organic particles up to $100 \mu \mathrm{m}$ in diameter and mineral particles a few $\mu \mathrm{m}$ in diameter (clay) may be resuspended. These conclusions are consistent with the observed particle mass. Particle resuspension most probably occur sporadically, whereas particle redistribution within the BBL is accomplished by long term seiche-induced mixing.

\section{Summary and conclusions}

1. Since the well stratified hypolimnion of Lake Alpnach was shielded from the direct influence of the wind, bottom currents (measured ca $1 \mathrm{~m}$ above the sediment) were solely the result of isotherm motion. Both the first and second vertical seiche modes were excited.

2. The internal seiching motion was highly correlated to the bottom currents, which had an average speed of $c a 2 \mathrm{~cm} \mathrm{~s}^{-1}$. During highfrequency burstlike events current speeds of up to $7 \mathrm{~cm} \mathrm{~s}^{-1}$ occurred. 
3. The internal seiche-induced bottom currents established a distinct and quasi-steady-state well-mixed BBL with a temporally varying thickness of 2 to $7 \mathrm{~m}$. Temperature gradients within this layer were an order of magnitude smaller than just above it. Since more than a month is required to build up a BBL of such a magnitude, we conclude that the changing thickness was only associated with the seiching motion and not with erosion of the stability repetitively induced by boundary mixing.

4. Resuspension was indicated by the vertical distribution of suspended particles. Within the BBL the particle concentration was always about three times greater than above. The maximum concentration was found near the sediment, where turbulent diffusion dominates over particle settling.

5. $95 \%$ of the volume of the particles within the BBL were found to be of organic origin. According to the extended Shields diagram (Fig. 6), the observed currents with a mean speed of about $2 \mathrm{~cm} \mathrm{~s}^{-1}$ would be too weak to initiate motion of inorganic sediment particles.

6. Resuspension is thought to be associated with high-frequency burst-like currents with measured speeds ranging up to $7 \mathrm{~cm} \mathrm{~s}^{-1}$ rather than with the average bottom current speed of about $2 \mathrm{~cm} \mathrm{~s}^{-1}$. In situ bottom currents of this magnitude have been reported to initiate resuspension (Lemmin \& Imboden, 1987).

7. The phenomenon of intense internal seiching can therefore be expected to be a relevant source of resuspension in many lakes.

\section{Acknowledgements}

We are indebted to A. Zwyssig and A. Vagenknecht for the laboratory work, to F. Peeters and M. Schurter for support in the field, to N. H. Brooks for helpful discussions on resuspension and to D.M. Livingstone for interpreting the Shields diagram and improving the English. This work was supported by Swiss National Science Foundation grant 20-32700.91.

\section{References}

Baker, E. T. \& J. W. Lavelle, 1984. The effect of particle size on the Light attenuation coefficient of natural suspensions. J. Geophys. Res. 89: 8197-8203.

Bloesch, J. \& U. Uehlinger, 1986. Horizontal sedimentation differences in a eutrophic Swiss lake. Limnol. Oceanogr. 31: 1094-1109.

Dewey, R. K. \& W. R. Crawford, 1988. Bottom stress estimates from vertical dissipation rate profiles on the continental shelf. J. Phys. Oceanogr. 18: 1167-1177.

Elliott, A. J., 1984. Mcasurements of the turbulence in an abyssal boundary layer. J. Phys. Oceanogr. 14: 1778-1786.

Imboden, D. M. \& A. Wüest, 1994. Mixing mechanisms in Lakes. (to be published in 'Lakes' (ed.), Springer).

Johnson, C. A., M. Ulrich, L. Sigg \& D. M. Imboden, 1991. A mathematical model of the manganese cycle in a seasonally anoxic lake. Limnol. Oceanogr, 36: 1415-1426.

Jørgenssen, B. B. \& D. J. Des Marais, 1990. The diffusive boundary layers of sediments: Oxygen microgradients over a microbial mat. Limnol. Oceanogr. 35: 1343-1355.

LaZerte, B. D., 1980. The dominating higher order vertical modes of the internal seiche in a small lake. Limnol. Oceanogr. 25: 846-854.

Lemmin, U. \& D. M. Imboden, 1987. Dynamics of bottom currents in a small lake. Limnol. Oceanogr. 32: 62-75.

Miller, M. C., I. N. McCave \& P. D. Komar, 1977. Threshold of sediment motion under unidirectional currents. Sedimentology 24: 507-527.

Münnich, M., A. Wüest \& D. M. Imboden, 1993. Observations of the second vertical mode of the internal seiche in an alpine lake. Limnol. Oceanogr. 37: 1705-1719.

Pak, H. \& J. R. Zaneveld, 1983. Temporal variations of beam attenuation coefficient on the continental rise off Nova Scotia. J. Geophys. Res. 88: 4427-4432.

Sandilands, R. G. \& A. Mudroch, 1983. Nepheloid Layer in Lake Ontario. J. Great Lakes, 9: 190-200.

Shields, A. 1936. Anwendung der Aehnlichkeitsmechanik und der Turbulenzforschung auf die Geschiebebewegung. Mitt. preuss. Versuchsanst. Wasserbau Schiffbau, Berlin 26: $1-26$.

Thorpe, S. A., 1988. The dynamics of the boundary layers of the deep ocean. Sci. Prog., 72: 189-206.

Vanoni, V. A., 1964. Measurements of critical shear stress for entraining fine sediments in a boundary layer. Report No. KH-R-7, W. M. Keck Laboratory Caltech, Pasadena, California.

Wehrli, B., G. Friedl \& A. Manceau, 1993. Reaction rates and products of manganese oxidation at the sediment-water interface. In Huang, C. P., C. O'Melia \& J. J. Morgan (eds), Aquatic Chemistry. ACS Advances in Chemistry, Washington DC, Series 244 (in press).

Wiegand, R. C. \& V. Chamberlain, 1987. Internal waves of the second vertical mode in a stratified lake. Limnol. Oceanogr. 32: 29-42.

Yalin, M. S, 1977. Mechanics of sediment transport. Pergamon Press. 